\title{
Atomic Friction Investigations on Ordered Superstructures
}

\author{
Pascal Steiner • Enrico Gnecco • Tobin Filleter • \\ Nitya Nand Gosvami $\cdot$ Sabine Maier • \\ Ernst Meyer $\cdot$ Roland Bennewitz
}

Received: 4 February 2010/Accepted: 22 July 2010/Published online: 7 August 2010

(C) Springer Science+Business Media, LLC 2010

\begin{abstract}
We review recent friction measurements on ordered superstructures performed by atomic force microscopy. In particular, we consider ultrathin $\mathrm{KBr}$ films on $\mathrm{NaCl}(001)$ and $\mathrm{Cu}(001)$ surfaces, single and bilayer graphene on $\mathrm{SiC}(0001)$, and the herringbone reconstruction of $\mathrm{Au}(111)$. Atomically resolved friction images of these systems show periodic features spanning across several unit cells. Although the physical mechanisms responsible for the formation of these superstructures are quite different, the experimental results can be interpreted within the same phenomenological framework. A comparison between experiments and modeling shows that, in the cases of $\mathrm{KBr}$ films on $\mathrm{NaCl}(001)$ and of graphene films, the tipsurface interaction is well described by a potential with the periodicity of the substrate which is modulated or, respectively, superimposed with a potential with the symmetry of the superstructure.
\end{abstract}

P. Steiner · E. Gnecco $(\bowtie) \cdot$ S. Maier · E. Meyer

Department of Physics, University of Basel,

Klingelbergstrasse 82, 4056 Basel, Switzerland

e-mail: enrico.gnecco@unibas.ch

T. Filleter

Department of Physics, McGill University, Montreal, QC H3A 2T8, Canada

N. N. Gosvami · R. Bennewitz

INM, Leibniz Institute for New Materials,

66123 Saarbrücken, Germany

Present Address:

S. Maier

Materials Science Division, Lawrence Berkeley National

Laboratory, Cyclotron Road 1, Berkeley, CA 94720, USA
Keywords Nanotribology · Friction mechanisms · Stick-slip · AFM

\section{Introduction}

Atomic force microscopy (AFM) is an invaluable technique to investigate friction forces on the nanometer scale [1]. When the probing tip of an AFM slides on a flat crystal surface in a clean environment such as an ultra-high vacuum, and the tip is sufficiently sharp, stick-slip motion across the unit cells of the sample can be easily observed [2]. Atomic stick-slip appears in time as a sawtooth signal reflecting the torsional deformation of the cantilever holding the tip. The periodicity of the stick-slip pattern usually corresponds to the unit cell of the surface lattice, although tip jumps of a few lattice constants can be detected [3, 4]. The cantilever torsion can be readily quantified if the AFM is equipped with a four-quadrant photodector. In such a case, the instrument can be operated as a friction force microscope (FFM). It is important to note that atomic stick-slip can be recognized also with blunt tips or even when sample material is worn off and transferred to the tip apex [5]. The only requirement in such cases is that a commensurable contact between tip and surface is established. This can be achieved via a reorganization of the atomic layers transferred from sample to tip [6] or via sudden rotations of flakes picked up from the substrate in the case of layered materials [7]. If a commensurate contact is not formed, a regime of ultralow friction-so-called 'structural lubricity' - may be observed $[8,9]$. Ultralow friction can also be experienced with sharp tips, provided that the normal load is reduced below a certain 'superlubric' threshold [10, 11]. 
Topography and friction maps of atomically flat crystal surfaces are necessarily convoluted with the atomic structure of the tip apex. This makes the resolution limits of AFM in contact mode quite difficult to determine, and friction imaging a complex process to model. A better understanding could be gained by imaging atomic-scale impurities or nanostructures. However, although atomic defects can be revealed in non-contact AFM (NC-AFM) [12], and even in contact when mechanical resonances are tracked [13], this is usually not the case in lateral force images of flat surfaces. Larger structures formed by annealing or irradiating materials like alkali halides are neither good candidates to test the ultimate resolution of FFM, since step edges and corner sites are prone to be worn off, due to the lower coordination of the atoms at these sites [14]. More stable patterns, eventually formed by different materials, are desirable. In this context, quite interesting structures can be realized by the heteroepitaxial growth of thin films. For instance, it is well established that Moiré patterns can be observed by STM on ultrathin alkali halide films on metal single crystals $[15,16]$. Superstructures can also be observed when a thin film is grown on a reconstructed surface. This is the case of graphene on SiC, as also demonstrated by STM [17, 18, 19]. However, whether the superstructure observed by STM has an influence on mechanical properties like friction on the adsorbate films cannot be revealed by STM alone.

The goal of this paper is to discuss recent studies that demonstrated the observation of ordered superstructures on atomically flat substrates by FFM and revealed tribological properties of these structures down to the atomic scale. In particular, we focus our attention on thin alkali halide films deposited on single crystals of another alkali halide or a metal surface, on graphene overlayers formed on $\mathrm{SiC}(0001)$, and on the herringbone reconstruction of $\mathrm{Au}(111)$. Experimental results are reproduced modelling the tip-surface interaction with phenomenological potentials, which extend the classical Prandtl-Tomlinson model used to interpret friction on atomically flat surfaces $[20,21]$ to the superstructures. The importance of these studies goes beyond understanding the mechanisms and testing the resolution limits of FFM. The investigation of the $\mathrm{KBr} / \mathrm{NaCl}(001)$ system, for instance, clearly revealed how both the periodicity of the overlayers and the superperiodicity of the 'buried' $\mathrm{KBr} / \mathrm{NaCl}$ interface have a comparable influence on the motion of the tip sliding on the top of the composite system.

We should mention that atomically ordered superstructures were observed by AFM also on other systems (without friction maps). In the past decade hexagonal superstructures were discerned in intercalation compounds of the layered $\mathrm{MnPS}_{3}$ and associated with protruding sulfur atoms [22]. Incommensurate superstructures were recognized on insulating layered material and attributed to a phase transition in the perovskite structure of the crystal [23], and also on conducting surfaces, where they were related to charged density waves [24].

\section{Experimental results}

Since the lattice constants of $\mathrm{NaCl}(0.565 \mathrm{~nm})$ and $\mathrm{KBr}$ $(0.660 \mathrm{~nm})$ are almost in a ratio 6:7, a significant rearrangement of the ions at the interface is expected when one of the two alkali halides is deposited on the second one. Recent NC-AFM studies pointed out important differences between $\mathrm{NaCl} / \mathrm{KBr}(001)$ and the 'reciprocal' system, i.e. $\mathrm{KBr} / \mathrm{NaCl}(001)$ [25]. In the first case the ionic bonds are allowed a considerable relaxation and the overlayer stretches over the substrate to match its lattice structure. This is not the case when $\mathrm{KBr}$ is deposited on $\mathrm{NaCl}$, and a 'rumpling' of the interface is energetically preferred to a compression of the $\mathrm{K}-\mathrm{Br}$ bonds. The latter effect can be easily understood observing that ions with the same sign overlap with a periodicity of $7 \mathrm{NaCl}$ lattice constants so that the Coulomb repulsion increases the corrugation of the interface at the locations of these ions. The deformation of the interface will also affect the growth of $\mathrm{KBr}$ thin films up to a few monolayers, as suggested by Monte Carlo simulations [26]. While a single $\mathrm{KBr}$ layer could not be recognized in NC-AFM measurements, the rumpling was observed on 2- and 3-ML thick islands. The ions in the $\mathrm{KBr}$ top layer organized in a square superstructure whose unit cell covered $7 \times 7$ unit cells of the substrate. The apparent corrugation of the superstructure in NC-AFM topographies was about $0.12 \mathrm{~nm}$ on $2 \mathrm{ML}$ and $0.11 \mathrm{~nm}$ on 3-ML-thick islands [25]. The rumpling effect has been recently recognized also in topographies acquired in contact mode (not shown), and clear evidence of atomic stick-slip was given by the corresponding friction maps (Fig. 1a). Compared to the regular stick-slip on bulk-truncated $\mathrm{KBr}(001)$ surfaces, the stickslip ampitude on $2 \mathrm{ML} \mathrm{KBr} / \mathrm{NaCl}(001)$ is modulated with the periodicity of the superstructure and the offset oscillates with the same period. The modulation of the friction force is reduced on $3 \mathrm{ML}$ films, where the $\mathrm{KBr} / \mathrm{NaCl}$ interface has clearly less influence. If the normal load is lowered below a certain threshold (close to the cantilever jump-off) regular stick-slip is observed. In the section profiles in Fig. 1a, a slight variation of the slope can be also recognized. This slope is essentially associated with the lateral stiffness of the contact region [10], so that the question arises whether this quantity, rather than the tip-surface interaction, is more influenced by the superstructure. We will come back to this question in the next section.

In the case of $\mathrm{KBr}$ growing on $\mathrm{Cu}(001)$ the ratio between $\mathrm{Cu}(0.361 \mathrm{~nm})$ and $\mathrm{KBr}$ lattice constants is slightly larger than 6:11, which also results in the 

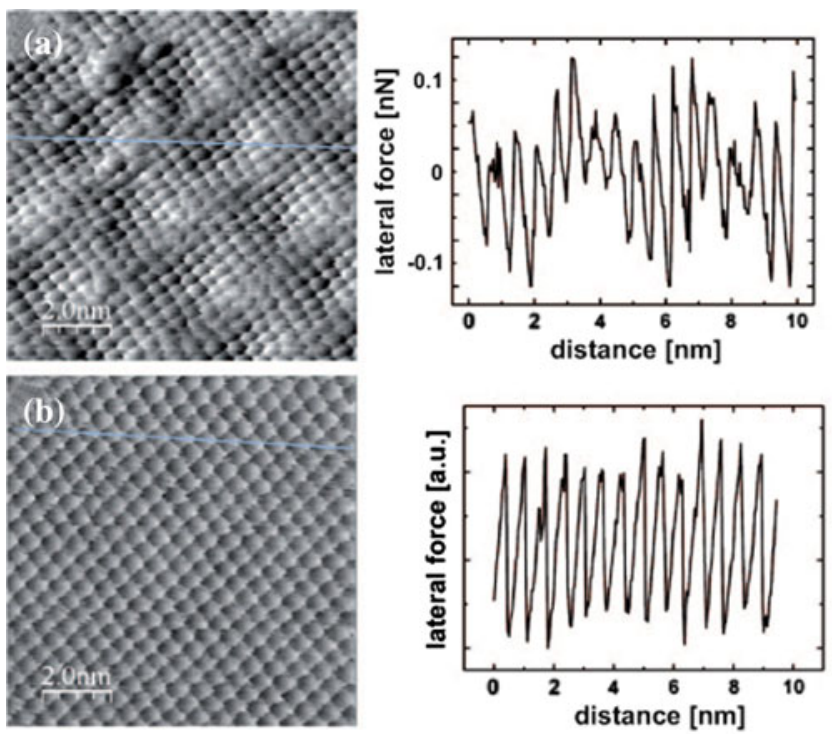

Fig. 1 Friction force maps experimentally acquired on a $\mathrm{KBr} /$ $\mathrm{NaCl}(001)$ (adapted from [27]), b $\mathrm{KBr} / \mathrm{Cu}(001)$, c graphene/ $\mathrm{SiC}(0001)$, and $\mathbf{d}$ the herringbone reconstruction of $\mathrm{Au}(111)$. Ordered

formation of regular superstructures. Since a rumpling effect caused by the repulsion of similar ionic species is excluded, the structuring will be essentially caused by the compression of $\mathrm{K}-\mathrm{Br}$ bonds. The presence of a square pattern with a periodicity of $3.96 \mathrm{~nm}$ was revealed in NC-AFM topographies on 2-, 3- and 4-ML-thick films [28]. A slight corrugation of about $30 \mathrm{pm}$ was also measured, which is in the same range of the atomic corrugation of the lattice. Friction force images acquired on the same system also show a very weak spatial modulation (Fig. 1b). The values of the friction force and lateral contact stiffness recorded on the $\mathrm{KBr}$ film are in the same range of bulktruncated $\operatorname{KBr}(001)$ surfaces.

The presence of a $6 \times 6$ superstructure on 1- and 2-MLthick graphene films grown on $\mathrm{SiC}(0001)$ using thermal decomposition under atmospheric pressure was recently revealed by NC-AFM [29]. This superstructure of graphene is following the $6 \times 6$ reconstruction of the substrate surface, a carbon-rich interface layer which develops in the thermal decomposition process. Atomically resolved friction maps show that the superstructure also appears as a modulation in the lateral force (Fig. 1c). However, compared to the films formed by $\mathrm{KBr}$ on $\mathrm{NaCl}(001)$ and $\mathrm{Cu}(001)$, a remarkable difference can be noticed. Whereas $\mathrm{KBr}$ films show an amplitude modulation of the friction force, the graphene layers present a periodic variation in the offset of the stick-slip pattern. Furthermore, the superstructure is maintained in the superlubric regime, which sets on at relatively high loads (around $40 \mathrm{nN}$ ).

Finally, we would like to present recent FFM measurements on the well-known herringbone reconstruction
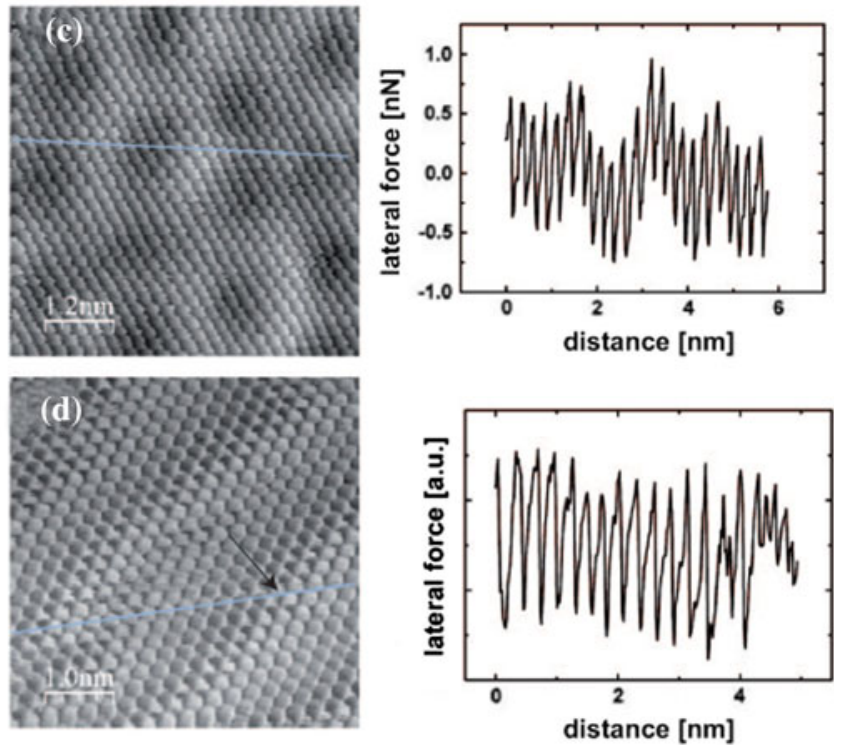

superstructures can be recognized in all systems. Cross sections taken along one of the principal crystallographic directions of the substrates are shown on the right side of the images

of the $\mathrm{Au}(111)$ surface [30]. Fig. 1d shows a lateral force map acquired at low load (in the $\mathrm{nN}$ range) on a flat area of this surface. The signature of the herringbone reconstruction is given by periodic shifts in the atomic rows, one of which is highlighted by the section. These shifts reflect a change of stacking across the surface, which does not allow extracting the modulation of stick-slip as it was done for the superstructures presented so far. On reconstructed $\mathrm{Au}(111)$ the average value of the friction force remained below the noise level for normal loads up to $3.8 \mathrm{nN}$ [31]. Above this value, friction suddenly increased and wear set on.

\section{Discussion}

Atomic friction on flat surfaces is well-interpreted by the Prandtl-Tomlinson model [20, 21] (or by its extension known as Frenkel-Kontorova-Tomlinson model in the case of extended contact areas [32]). In the Tomlinson model the probing tip is approximated by a point mass $m$ which is laterally driven by an elastic spring (with spring constant $k$ ) across a potential $V(x, y)$, describing the chemical interaction between the tip apex and the surface lattice. Here, we aim to extend this model to the ordered superstructures discussed in the previous section. The most striking observation in the experimental results is the different response of $\mathrm{KBr}$ films on $\mathrm{NaCl}(001)$ and graphene. On $\mathrm{KBr}$ films both amplitude and offset of stick-slip are modulated, whereas on graphene only the offset of the stick-slip oscillates with the periodicity of the superstructure. We have noticed that both behaviors can be 
well-reproduced if the tip-surface interaction potential $V(x, y)$ is written as a combination of two potentials: $V_{\text {lat }}(x, y)$, with the periodicity of the top layer, and $V_{\text {sup }}(x, y)$, with the periodicity of the superstructure. If we limit our analysis to the first Fourier components of $V$ and $V_{\text {sup }}$, these potentials can both be written as superpositions of two (for square symmetries) or three plain waves (for hexagonal symmetries) $e^{i \mathbf{k}} \cdot \mathbf{r}$, where the wave vector $\mathbf{k}$ and the radius $\mathbf{r}$ are oriented parallel to the substrate surface. These waves are tilted with respect to one another by $90^{\circ}$ in the case of $\mathrm{KBr}$ films and by $60^{\circ}$ in the case of graphene. Introducing the spatial periodicity $a$ of the atomic lattice and the periodicity $b$ of the superstructure, the wave numbers for the potentials $V_{\text {lat }}$ and $V_{\text {sup }}$ are $2 \pi / a$ and $2 \pi / b$, respectively.

We find a good agreement with the experimental results introducing the following assumptions:

a. In the case of $\mathrm{KBr} / \mathrm{NaCl}(001)$ we assume that the amplitude of the potential $V_{\text {lat }}$ is modulated by the 'superpotential' $V_{\text {sup }}$ :

$V(x, y)=V_{\text {lat }}\left(1+\alpha \frac{2 V_{\text {sup }}}{E_{0}}\right)$,

where $E_{0}$ is the corrugation amplitude of the potential $V_{\text {lat }}$ and the parameter $\alpha$ gives the strength of the modulation effect. Since the substrate has a square symmetry, the potential $V_{\text {lat }}$ can be written in the form

$V_{\text {lat }}(x, y)=-\frac{E_{0}}{2} \cos \frac{2 \pi x}{a} \cos \frac{2 \pi y}{a}$.

The potential $V_{\text {sup }}$ has also the form 2, with the lattice constant $a$ replaced by the period $b$ of the superstructure. The interaction potential 1 is shown in Fig. 2 a.

b. In the case of graphene, the experimental results are better reproduced by a superposition of two potentials $V_{\text {lat }}$ and $V_{\text {sup: }}$ :

$V(x, y)=V_{\text {lat }}+\beta \widehat{R} V_{\text {sup }}$,

where the parameter $\beta$ gives the ratio between the amplitudes of the superperiodic potential $V_{\text {sup }}$ and the atomic potential $V_{\text {lat }}$ and the operator $\widehat{R}$ describes a possible rotation of the coordinate system. Due to the hexagonal symmetry of the system, the potential $V_{\text {lat }}$ takes now the form

$V_{\text {lat }}(x, y)=-\frac{E_{0}}{4.5}\left(2 \cos \frac{2 \pi x}{a} \cos \frac{2 \pi y}{a \sqrt{3}}+\cos \frac{4 \pi y}{a \sqrt{3}}\right)$,

and similarly $V_{\text {sup }}$ (with the length $a$ replaced by $b$ ). Figure $2 \mathrm{~b}$ shows a possible interaction potential $V(x, y)$ with the form 3 .

The equations of motion of the tip, with its support moving at a speed $v$ in the $x$ direction, have consequently been solved in the two cases (a) and (b). Since the experiments were performed at room temperature, we have also introduced a noise term satisfying the fluctuation-dissipation theorem, and a damping term $-m \gamma\left(v_{x}, v_{y}\right)$, as usual in this kind of calculations [33]. The latter term guarantees the occurrence of atomic stick-slip provided that the parameters $k, m$ and $\gamma$ satisfy the relation $\gamma \gg \gamma_{\mathrm{cr}}=$ $2 \sqrt{k / m}$, i.e. that the oscillations of the tip, immediately after jumping, are overdamped [34]. Figure 3 shows numerical results obtained using the assumptions (a) and (b) respectively. In this figure, the lateral force $F_{x}=k(v t-x)$, as recorded along the fast scan direction, is mapped as a function of the support coordinates, as in the experiments. A satisfying agreement with the experimental data was obtained using the values (a) $E_{0}=1 \mathrm{eV}, k=2 \mathrm{~N} / \mathrm{m}$, and $\alpha=0.7$ for the $\mathrm{KBr}$ film and (b) $E_{0}=2 \mathrm{eV}, k=4.7 \mathrm{~N} / \mathrm{m}$, and $\beta=0.7$ for graphene. In the last case, the AFM data could be matched only after rotating the potential $V_{\text {sup }}$ by $30^{\circ}$ with respect to $V_{\text {lat }}$. We note that the rotation by $30^{\circ}$ between the graphene lattice and the $6 \times 6$ reconstruction was observed using STM and LEED [17].

With the choice of potential (1) we implicitly assumed that the tip-surface interaction is enhanced in the troughs of the superstructure. When the load is lowered or $\alpha$ decreases this effect is reduced till the interaction gets confined within the unit cell of the superstructure when $\alpha=0$. Another possibility is that the lateral stiffness rather than the interaction potential is spatially modulated. However, calculations performed under this hypothesis (shown in the 1D case [27]) are inconsistent with the experimental observations. This result gives a strong indication the $\mathrm{KBr} /$ $\mathrm{NaCl}$ interface plays an important role in determining the frictional response on the top layer. On the other side, it is difficult to rationalize the good matching between the assumption (b) and the frictional maps acquired on graphene. We can only postulate that the modulated offset of the lateral force originates in the fact that the substrate surface undergoes a significant reconstruction during the growth of the graphene film. The much smaller modulated offset which is observed for $\mathrm{KBr}$ on $\mathrm{NaCl}(001)$ in addition to the amplitude modulation is well reproduced in the simulations in Fig. 3b based on the model potential (1). We have repeated the simulations for various parameters and found that in general a modulation of the lateral force amplitude occurs only for model potential of type (1), i.e. with a modulated amplitude of the atomic potential. We conclude that there is a fundamental difference between the effective lateral potentials on the $\mathrm{KBr}$ and the graphene films. While for the $\mathrm{KBr}$ films the amplitude of the lateral atomic potential is modulated by the superstructure, it is not modulated but periodically offset in the case of graphene. The origin of this difference is not clear at this time, and explanation will probably require a simulation with realistic atomic potentials. 
Fig. 2 Tip-surface interaction potentials corresponding a to the expression 1 and $\mathbf{b}$ to the expression 3 introduced in the text. The parameters $\alpha$ and $\beta$ are both set equal to 0.7 . In $\mathbf{b}$ the superpotential $V_{\text {sup }}$ is rotated by $30^{\circ}$ with respect to the normal potential $V_{\text {lat }}$
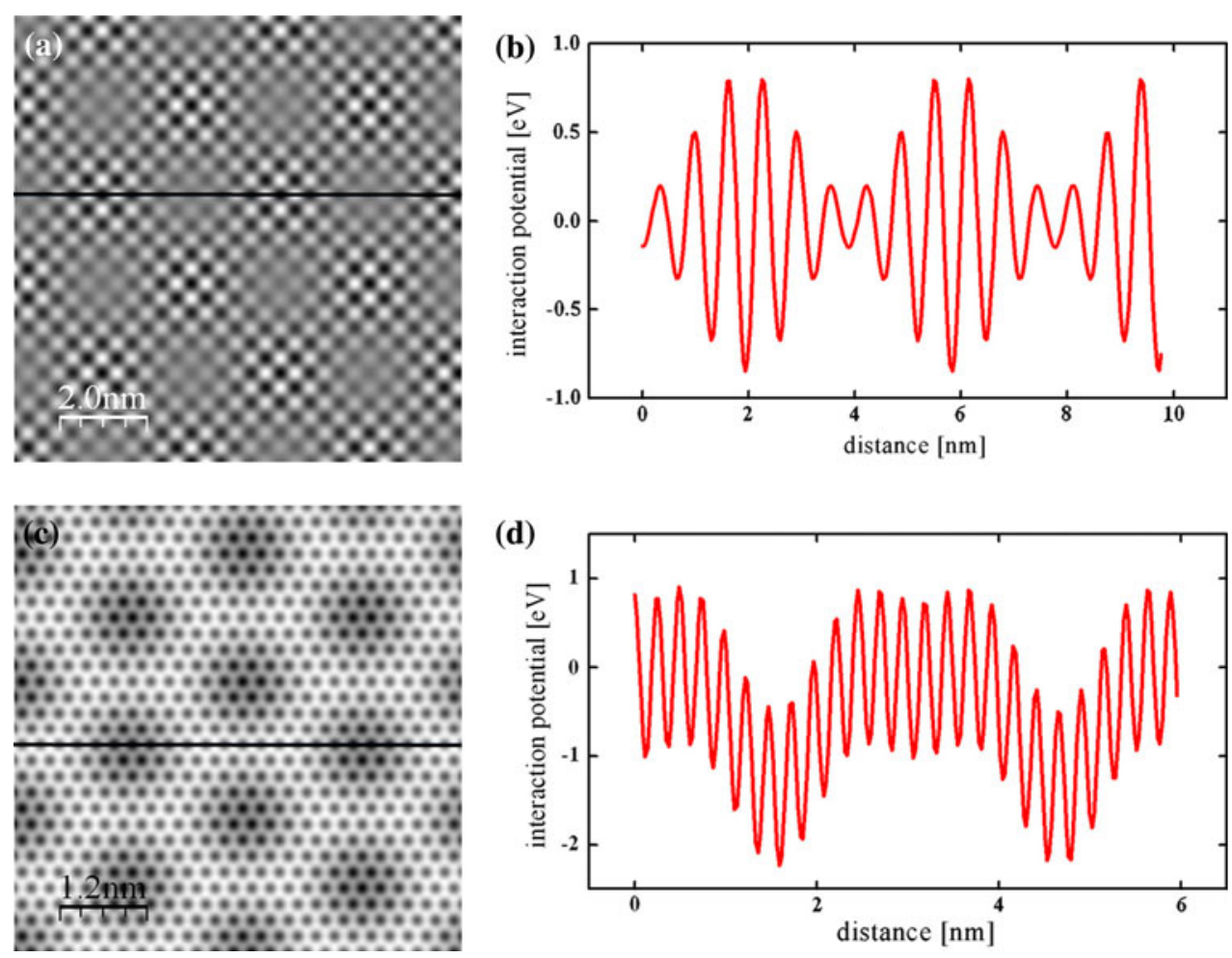

(d)

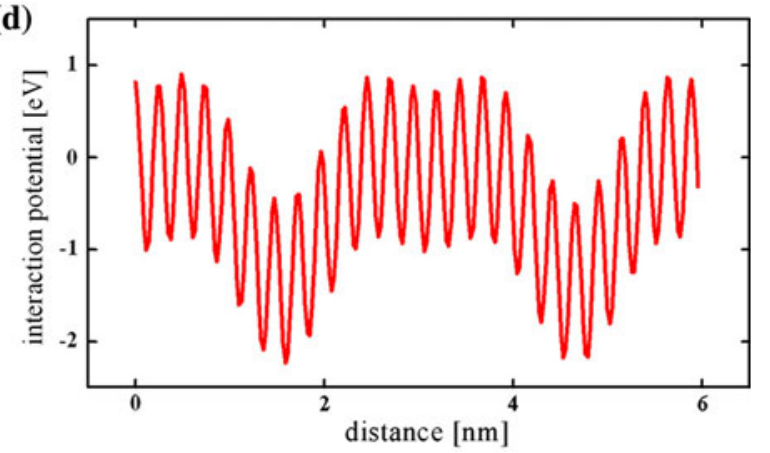

Fig. 3 Simulated friction maps corresponding to the potentials in Fig. 2. The values of scan speed, tip mass, and damping coefficient are respectively $v=25 \mathrm{~nm} / \mathrm{s}, m=10^{-12} \mathrm{~kg}$ and $\gamma=10 \gamma_{\mathrm{cr}}$
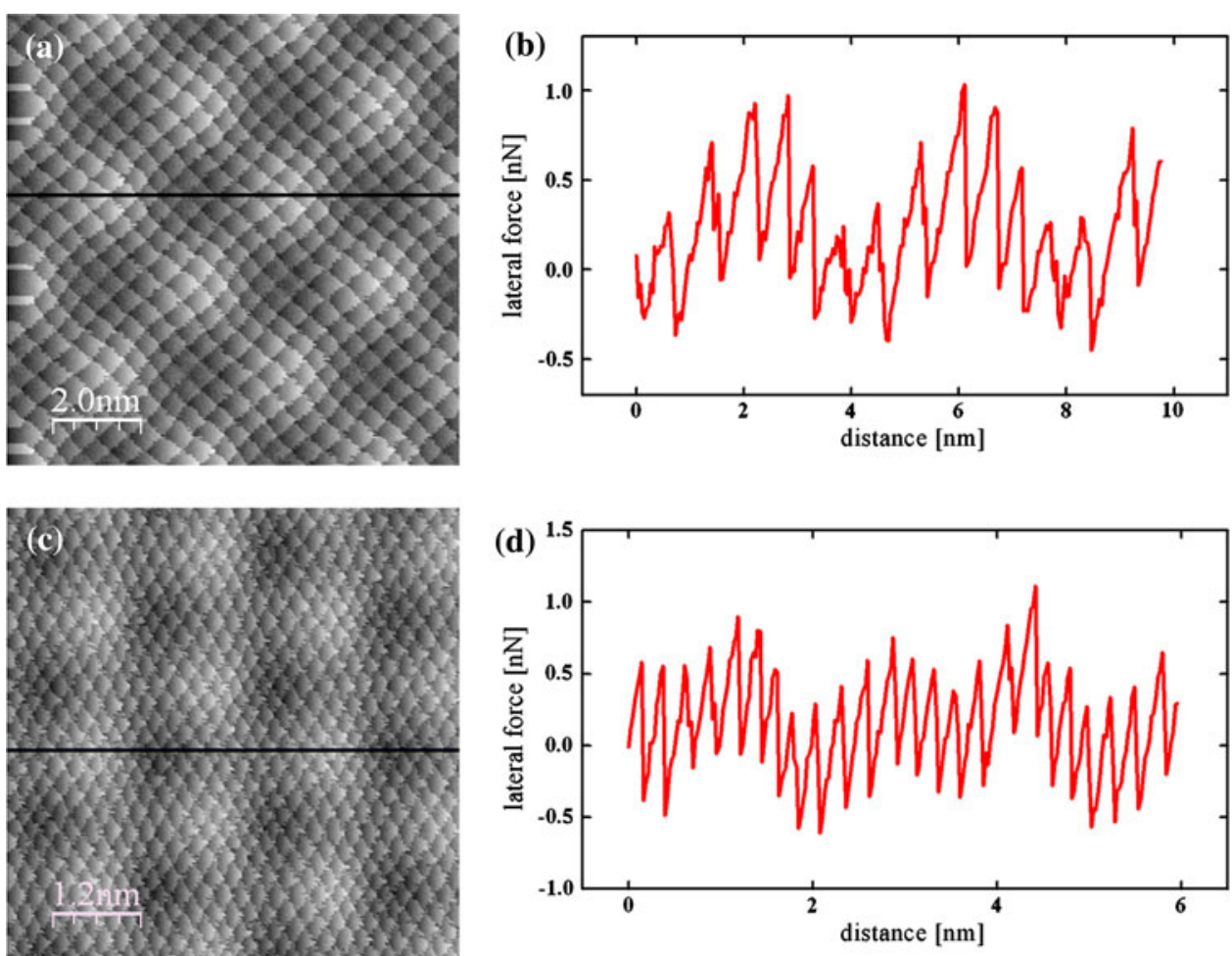

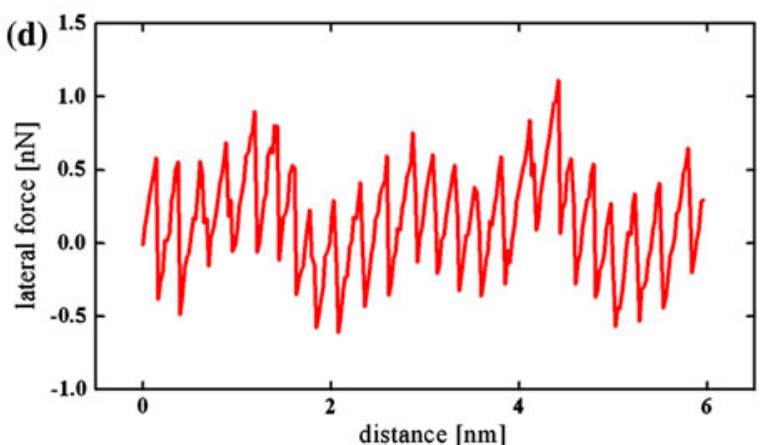

We have not attempted to reproduce the frictional response of the reconstructed $\mathrm{Au}(111)$ surface by periodically shifting the atomic rows. Here, we can only suggest that the tilt in the lateral force curve in Fig. 1d is caused by the facetting of the $\mathrm{Au}(111)$ surface due to the herringbone reconstruction. Furthermore, we would like to emphasize the details of the structure resolved in Fig. 1d. As shown by the arrow, the shift in the herringbone reconstruction can be localized within the spatial limit of a lattice constant. Similarly, Fig. 1a shows stable defects at well-defined 
atomic locations. Since these defects differ in both shape and size, they are not simply mirroring the atomic arrangement at the tip apex. This gives an answer to the question about the resolution limits of FFM which was stated in the introduction. Friction force microscopy is shown here to approach atomic resolution, provided that the probing tip is very sharp and one operates at very low loads (close to the superlubric regime [10]). We have indeed indications that the corrugation of the superstructure as observed in lateral force maps becomes smaller (and defects disappear) using blunter tips.

\section{Conclusion and Outlook}

We have discussed four recent examples of frictional imaging on atomically ordered superstructures. Two of them $[\mathrm{KBr}$ on $\mathrm{NaCl}(001)$ and graphene on $\mathrm{SiC}(0001)]$ could be well reproduced invoking the Prandtl-Tomlinson model and using appropriate combinations of two periodic potentials to describe the tip-surface interaction. The amplitude of the atomic potential is modulated by the superstructure in the case of $\mathrm{KBr}$ films on $\mathrm{NaCl}(001)$, whereas the two potentials are superimposed and rotated in the case of graphene films. The form of the first potential is traced back to the rumpling of the buried $\mathrm{KBr} / \mathrm{NaCl}$ interface, whereas more detailed theoretical investigations should clarify the physical mechanisms responsible for the shape of the second potential. The friction maps presented in this paper also demonstrate the atomic scale resolution capabilities of FFM.

Frictional investigations on superstructures may be extended beyond the few examples discussed in this paper. A regular arrangement of ion impurities resulting in the socalled Suzuki phase was recently recognized by Barth and Henry [35] on alkali halide surfaces. Another noticeable system are boron nitride nanomeshes, whose structure seems to be quite resistant to external solicitations [36]. Quasicrystals are also attracting considerable interest in nanotribology [37] and it would be quite intriguing although challenging to determine whether superstructures can be grown on them and resolved by friction force microscopy.

Acknowledgements The Swiss National Science Foundation, the Swiss National Center of Competence in Research on Nanoscale Science, and the European Science Foundation (EUROCORES Programme FANAS) are gratefully acknowledged for financial support.

\section{References}

1. Bennewitz, R., Meyer, E., Hug H.-J.: Scanning Probe Microscopy. Springer, Berlin (2003)
2. Szlufarska, I., Chandross, M., Carpick, R.W.: Recent advances in single-asperity nanotribology J. Phys. D: Appl. Phys. 41, 123001 (2008)

3. Medyanik, S.N., Liu, W.K., Sung, I.H., Carpick, R.W.: Predictions and observations of multiple slip modes in atomic-scale friction. Phys. Rev. Lett. 97, 136106 (2006)

4. Roth, R., Glatzel, T., Steiner, P., Gnecco, E., Baratoff, A., Meyer, E.: Multiple slips in atomic-scale friction: an indicator for the lateral contact damping. Trib. Lett. 39, 63-69 (2010)

5. Gnecco, E., Bennewitz, R., Meyer, E.: Abrasive wear on the atomic scale. Phys. Rev. Lett. 91, 215501 (2002)

6. Livshits, A.I., Shluger, A.L.: Self-lubrication in scanning-forcemicroscope image formation on ionic surfaces. Phys. Rev. B 56, 12482-12489 (1997)

7. Filippov, A.E., Dienwiebel, M., Frenken, J.W.M., Klafter, J., Urbakh, M.: Torque and twist against superlubricity. Phys. Rev. Lett. 100, 046102 (2008)

8. Dienwiebel, M., Verhoeven, G.S., Pradeep, N., Frenken, J.W.M., Heimberg, J.A., Zandbergen, H.W.: Superlubricity of graphite. Phys. Rev. Lett. 92, 126101 (2004)

9. Müser, M.: Structural lubricity: role of dimension and symmetry. Europhys. Lett. 66,97-103 (2004)

10. Socoliuc, A., Bennewitz, R., Gnecco, E., Meyer, E.: Transition from stick-slip to continuous sliding in atomic friction: entering a new regime of ultra-low friction. Phys. Rev. Lett. 92, 134301 (2004)

11. Gnecco, E., Maier, S., Meyer, E.: Superlubricity of dry nanocontacts. J. Phys. Condens. Matt. 20, 354004 (2008)

12. Bammerlin, M., Lüthi, R., Meyer, E., Baratoff, A., Lü, J., Guggisberg, M., Loppacher, C., Gerber, C., Güntherodt, H.-J.: Dynamic SFM with true atomic resolution on alkali halide surfaces. Appl. Phys. A 66, S293-S294 (1998)

13. Steiner, P., Roth, R., Gnecco, E., Glatzel, Th., Baratoff, A., Meyer, E.: Modulation of contact resonance frequency accompanying atomic-scale stick-slip in friction microscopy. Nanotechnology 20, 495701 (2009)

14. Socoliuc, A., Gnecco, E., Bennewitz, R., Meyer, E.: Ripple formation induced in localized abrasion. Phys. Rev. B 68, 115416 (2003)

15. Pivetta, M., Patthey, F., Stengel, M., Baldereschi, A., Schneider, W.D.: Local work function Moiré pattern on ultrathin ionic films: $\mathrm{NaCl}$ on $\mathrm{Ag}(100)$. Phys. Rev. B 72, 115404 (2005)

16. Repp, J., Meyer, G., Rieder, K.H.: Snell's law for surface electrons: refraction of an electron gas imaged in real space. Phys. Rev. Lett. 92, 036803 (2004)

17. Riedl, C., Starke, U., Bernhardt, J., Franke, M., Heinz, K.: Structural properties of the graphene-SiC(0001) interface as a key for the preparation of homogeneous large-terrace graphene surfaces. Phys. Rev. B 76, 245406 (2007)

18. Mallet, P., Varchon, F., Naud, C., Magaud, L., Berger, C., Veuillen, J.Y.: Electron states of mono- and bilayer graphene on $\mathrm{SiC}$ probed by scanning-tunneling microscopy. Phys. Rev. B 76, 041403 (2007)

19. Rutter, G.M., Crain, J.N., Guisinger, N.P., Li, T., First, P.N., Stroscio, J.A.: Scattering and interference in epitaxial graphene. Science 317, 219-222 (2007)

20. Prandtl, L., Angew, Z.: Mind model of the kinetic theory of solid bodies. Math. Mech. 8, 85-106 (1928)

21. Tomlinson, G.A.: A molecular theory of friction. Philos. Mag. 7, 905-939 (1929)

22. Lagadic, I., Clement, R., Kahn, O., Ren, J., Whangbo, M.H.: Atomic-force microscopy study of layered $\mathrm{MnPS}_{3}$ and its intercalation compounds. Chem. Mat. 6, 1940-1945 (1994)

23. Kityk, A.V., Musevic, I., Slak, G., Fuith, A., Blinc, R.: Observation of an incommensurately modulated structure in dielectrics by an atomic-force microscope. Europhys. Lett. 36, 373-378 (1996) 
24. Slough, C.G., McNairy, W.W., Coleman, R.V., Garnaes, J., Prater, C.B., Hansma, P.K.: Atomic force microscopy and scanning tunneling microscopy of charge-density waves in 1T-TaSe2 and 1T-TaS2. Phys. Rev. B 42, 9255-9258 (1990)

25. Maier, S., Pfeiffer, O., Glatzel, Th., Meyer, E., Filleter, T., Bennewitz, R.: Asymmetry in the reciprocal epitaxy of $\mathrm{NaCl}$ and KBr. Phys. Rev. B 75, 195408 (2007)

26. Baker, J., Lindgard, P.A.: Monte Carlo determination of heteroepitaxial misfit structures. Phys. Rev. B 54, R11137-R11140 (1999)

27. Maier, S., Gnecco, E., Baratoff, A., Bennewitz, R., Meyer, E.: Atomic-scale friction modulated by a buried interface: combined atomic and friction force microscopy experiments. Phys. Rev. B 78, 045432 (2008)

28. Filleter, T., Paul, W., Bennewitz, R.: Atomic structure and friction of ultrathin films of $\mathrm{KBr}$ on $\mathrm{Cu}(100)$. Phys. Rev. B 77, 035430 (2008)

29. Filleter, T., Emtsev, K.V., Seyller, Th., Bennewitz, R.: Local work function measurements of epitaxial graphene. Appl. Phys. Lett. 93, 133117 (2009)

30. Wöll, C., Chiang, S., Wilson, R.J., Lippel, P.H.: Determination of atom positions at stacking-fault dislocations on $\mathrm{Au}(111)$ by scanning tunneling microscopy. Phys. Rev. B 39, 7988 (1989)
31. Gosvami, N.N., Filleter, T., Egberts, P., Bennewitz, R.: Microscopic friction studies on metal surfaces. Trib. Lett. 39, 19-24 (2010)

32. Weiss, M., Elmer, F.-J.: Dry friction in the Frenkel-KontorovaTomlinson model: static properties. Phys. Rev. B 53, 7539-7549 (1996)

33. Steiner, P., Roth, R., Gnecco, E., Baratoff, A., Maier, S., Glatzel, Th., Meyer, E.: Two-dimensional simulation of superlubricity on $\mathrm{NaCl}$ and highly oriented pyrolytic graphite. Phys. Rev. B 79, 045414 (2009)

34. Johnson, K.L., Woodhouse, J.: Stick-slip motion in the atomic force microscope. Trib. Lett. 5, 155-160 (1998)

35. Barth, C., Henry, C.R.: Imaging Suzuki precipitates on $\mathrm{NaCl}$ : $\mathrm{Mg}^{2+}(001)$ by scanning force microscopy. Phys. Rev. Lett. 100, 096101 (2008)

36. Corso, M., Auwärter, W., Muntwiler, M., Tamai, A., Greber, T., Osterwalder, J.: Boron nitride nanomesh. Science 303, 217-220 (2004)

37. Park, J.Y., Ogletree, D.F., Salmeron, M., Ribeiro, R.A., Canfield, P.C., Jenks, C.J., Thiel, P.A.: High frictional anisotropy of periodic and aperiodic directions on a quasicrystal surface. Science 309, 1354-1356 (2005) 